\title{
Los estudios sensoriales y la Edad Media: planteos historiográficos, desafíos y proyecciones
}

Gisela Coronado Schwindt

Universidad Nacional de Mar del Plata - CONICET

coronadogisela@gmail.com - https://orcid.org/0000-0001-8858-0406

Fecha recepción: 18.06.2019/ Fecha aceptación: 20.01.2020

\section{Resumen}

En los últimos años, investigadores de diversos campos disciplinares se propusieron comprender $y$ explicar el modo en que las percepciones sensoriales intervenían en la configuración de la dinámica social a lo largo del tiempo. En el plano historiográfico, la Historia de los sentidos permite realizar una nueva lectura de la documentación disponible, estudiando para cada época los registros sensoriales que transmiten las fuentes y la red de relaciones diná-

\begin{abstract}
In the last few years, researchers from various disciplinary fields have set out to understand and explain how sensory perceptions have intervened in shaping social dynamics over time. On a historiographical level, a history of the senses facilitates a new reading of the available documentation, studying the sensory perceptions recorded in the sources for each period and the network of dynamic relationships into which they are inserted.
\end{abstract}

\footnotetext{
* Este artículo ha sido realizado gracias a la participación de la autora en el proyecto de investigación "La Edad Media a través de los sentidos (I)" Subsidio HUM639/19 código de incentivo 15/F701, financiado por la Universidad Nacional de Mar del plata, República Argentina.
} 
micas en las que se insertan. Esta perspectiva no es simplemente un intento por reconstruir la variedad de percepciones sensoriales y su transformación de un período a otro o de una cultura a otra, sino que busca establecer la íntima conexión que existe entre una formación sensorial y los modos en que contribuye a interpretar la realidad y codificarla.

En este trabajo, nuestro objetivo general será analizar la conformación del campo de los estudios sensoriales y, en particular, abordaremos el desarrollo de la Historia de los sentidos en la Edad Media. Esto nos permitirá reconstruir la trayectoria de un problema historiográfico destacando las contribuciones teóricas y metodológicas desarrolladas por diversos autores. El propósito enunciado supone realizar una puesta a punto de los avances de esta perspectiva analítica que se proyecta en el tiempo, no exenta de dificultades y de peligros, despertando el ingenio de quienes asumen el desafío de traducir las marcas sensoriales que están impresas en todo registro material del pasado. En efecto, consideramos que la Historia de los sentidos se consolida como una línea de investigación de peso propio dentro del mundo académico que tiene una metodología particular, una vasta variedad publicaciones y una comunidad internacional activa de especialistas que recuperan los valores de la interdisciplinariedad y el trabajo colaborativo.

\section{Palabras clave}

Edad Media - Historia de los sentidos - Historiografía
This approach not only attempts to reconstruct the variety of sensory perceptions and how they change from to one period to another or from one culture from another, but also seeks to establish the intimate connection that exists between sensory formation and the ways in which this contributes to interpret and decode reality.

In this article, my general goal will be to analyse the configuration of the field of sensory studies, and in particular, I shall address the development of a sensory history of the Middle Ages. This will enable a reconstruction of the trajectory of a historiographical question by highlighting theoretical and methodological contributions by various authors. My intention is to provide an update on the progress of this analytical perspective over time, which although not without its difficulties and dangers, has awakened the ingenuity of those who take on the challenge of interpreting the sensory imprints that have left their mark on all records of the past. Indeed, I contend that sensory history is a consolidated area of research in the academic world, with a particular methodology, a wide variety of publications and an active international community of scholars who have revived the values of interdisciplinary and collaborative work.

\section{Keywords}

Middle Ages - Sensory History - Historiography 


\section{Introducción}

La ciencia histórica ha demostrado un interés constante por explorar nuevos campos de estudio que abarquen y comprendan las múltiples manifestaciones culturales ${ }^{1}$ de las sociedades a lo largo del tiempo ${ }^{2}$. Los historiadores, poco a poco, reconocieron que los sentidos, además de ser dotaciones corpóreas, poseen una importancia decisiva en el modo en que las personas perciben su mundo, constituyéndose en una vía de acceso a las elaboraciones perceptivas e intelectuales de la cultura. En opinión de Martín Jay, lo interesante de este tipo de abordajes «es, por supuesto, su compleja interacción a lo largo del tiempo lo que nos permite comprender que ni el sentido de los sentidos ni el sentido producido por los sentidos es invariable» ${ }^{3}$. La intención de posicionar a las percepciones sensoriales como medios válidos de conocimiento para las ciencias sociales ${ }^{4}$, originó la construcción de los llamados "estudios sensoriales" que tienen en la historiografía un desarrollo propio conocido como "Historia de los sentidos". Este campo ha estimulado la elaboración de un enfo-

1. En palabras de Peter Burke: "We seem to be living in an age of "turns", in historical thought and beyond. Famous turns such as the social turn, the linguistic turn and the cultural turn, all located in the later 20th century, have been followed more recently by the global turn, the affective or emotional turn, the material turn, the cognitive turn, the spatial turn, and a number of others» (Parece que estamos viviendo en una era de "giros", en el pensamiento histórico y más allá. Los giros famosos como el giro social, el giro lingüístico y el cultural, todos ubicados a fines del siglo XX, han sido seguidos más recientemente por el giro global, el giro afectivo o emocional, el giro material, el giro cognitivo, el espacial a su vez, $y$ una serie de otros), P. Burke, "Writing history in the $21^{\text {st }}$ century: challenges and responses", Historia 396, 8, 2, 2018, 13. Traducción nuestra.

2. J. Serna y A. Pons, La Historia Cultural. Autores, obras, lugares, Madrid, 2013, 5-14; P. Burke, ¿Qué es la historia cultura?, Barcelona, 2006; J. P. Rioux y J. F. Sirinelli, Para una Historia Cultural, Madrid, 1997, 1-23.

3. «It is, of course, their complex interaction over time that allows us to understand that neither the sense of the senses nor the sense produced by the senses is invariant», M. Jay, "The Senses in History. In the Realm of the Senses: An Introduction”, The American Historical Review, 116, 2, 2011, 307.

4. D. Howes, "Senses, Anthropology of the", en J. D. Wright (Ed.), International Encyclopedia of the Social \& Behavioral Sciences, Vol. 21, Oxford, 2015, 615-620. 
que multidisciplinario, planteando un «uso sistemático de los sentidos como herramienta metodológica y/o como objeto de estudio y reflexión» ${ }^{5}$.

La Historia de los sentidos permite realizar una nueva lectura de la documentación disponible, estudiando para cada época los registros sensoriales que transmiten las fuentes y la red de relaciones dinámicas en las que se insertan. Esta perspectiva no es simplemente un intento por reconstruir la variedad de percepciones sensoriales y su transformación de un período a otro o de una cultura a otra, sino que busca establecer la íntima conexión que existe entre una formación sensorial y los modos en que contribuye a interpretar la realidad y codificarla ${ }^{6}$. Se trata ante todo, en opinión de Daniela Hacke y Paul Musselwhite, de un amplio marco de análisis «porque los sentidos pueden interactuar y surgir de contextos diferentes y abarcar múltiples significados; los modos de percepción sensorial que se cruzan pueden superponerse y vincularse a construcciones culturales, políticas y sociales particulares, generando lo que los académicos han denominado "paisajes sensoriales" ${ }^{7}$.

En este trabajo, nuestro objetivo general será analizar la conformación del campo de los estudios sensoriales y, en particular, abordaremos el desarrollo de la Historia de los sentidos en la época medieval. Esto nos permitirá reconstruir la trayectoria de un problema historiográfico destacando las contribuciones teóricas y metodológicas desarrolladas por diversos autores. El propósito enunciado supone realizar una puesta a punto de los avances de esta perspectiva analítica que se proyecta en el tiempo, no exenta de dificultades y de peligros, despertando el ingenio de quienes asumen el desafío de traducir las marcas sensoriales que están impresas en todo registro material del pasado. En efecto, sostendremos que la Historia de los sentidos se consolida como una línea de investigación de peso propio dentro del mundo académico que tiene una metodología particular, una vasta variedad publicaciones y una comunidad internacional activa de especialistas que recuperan los valores de la interdisciplinariedad y el trabajo colaborativo.

\section{Precursores y constructores del campo sensorial en la historia y las ciencias sociales}

A fines del siglo XIX, los estudios sobre los sentimientos y las emociones de los sujetos comenzaron a ser considerados como una posible vía de exploración a raiz de los procesos y

5. A. L. Domínguez y A. Zirión, "Introducción al estudio de los sentidos”, en A. L. Domínguez y A. Zirión (Coord.), La dimensión sensorial de la cultura. Diez contribuciones al estudio de los sentidos en México, México, 2017, 9.

6. C. Classen, Worlds of Sense: Exploring the Senses in History and across Cultures, London, 1993, 50.

7. «Because senses can interact and arise from distinctly different contexts and cover multiple meanings; intersecting modes of sensory perception can overlap and become bound to particular cultural, political, and social constructs - generating what scholars have termed "sensescapes"», D. Hacke y P. Musselwhite, "Introduction: Making Sense of Colonial Encounters and New Worlds", en D. Hacke y P. Musselwhite (ed.), Empire of the Senses. Sensory Practices of Colonialism in Early America, Leiden-Boston, 2017, 9. 
acontecimientos sociales y políticos acaecidos a finales de la citada centuria y comienzos de la siguiente ${ }^{8}$. El inicio de la Gran Guerra y la crisis que produjo la pugna colonialista pusieron en tensión la idea del progreso indefinido de la historia ${ }^{9}$ y esto se tradujo en un cambio en la filosofía de las ciencias sociales. Estos procesos promovieron la elaboración de originales concepciones epistemológicas y la formulación de nuevas preguntas sobre las sociedades pasadas. Con esa ampliación llegó la necesidad de recurrir a otros registros más allá de los documentos producidos en el seno del poder. La multiplicación de los temas de investigación hizo necesaria la búsqueda de herramientas analíticas y conceptuales en otras ciencias sociales para comprender problemas de diversa índole.

En el marco de estos cambios, Johan Huizinga fue uno de los primeros historiadores en alertar sobre el perjuicio que representaba buscar sólo en los documentos oficiales los indicios necesarios para la construcción de la narrativa histórica, desestimando el empleo de otras fuentes que permitirían la comprensión de los distintos «tonos de vida». Su interés se centró en las distintas apariencias sensitivas de la sociedad del medioevo y el Renacimiento ${ }^{10}$, las cuales le posibilitaron construir un discurso sobre la sensibilidad en la Baja Edad Media. Para el autor, la extrema sensibilidad de esta época alcanzó su grado máximo en el arte rena-

8. J. Plamper, The History of Emotions. An Introduction, Oxford, Oxford University Press, 2015, 8. Los historiadores europeos destacaron los componentes emocionales en las ideas románticas sobre las "naciones", caracterizadas cada una de ellas con una forma colectiva de sentimiento, J. Plamper, "L'histoire des émotions", en C. Granger (Ed.), À quoi pensent les historiens? Faire de l'histoire au XXIe siècle, Paris, 2013, 225-249.

9. En los inicios del siglo XIX, la Historia comenzó el camino hacia su profesionalización, colocándose a la par de las ciencias duras y experimentales. Su concepción de los tiempos pasados se centró en una historia razonada y documentada, desprendida de toda subjetividad del historiador, girando en torno a la búsqueda exhaustiva de documentos, materiales a los que era necesario someter a un proceso de verificación, autentificación y comparación, bases epistemológicas y metodológicas de la narración histórica, E. Moradiellos, El oficio del historiador, Madrid, 1994, 33. Esta noción empirista de la práctica historiográfica, cuyos iniciadores más reconocidos fueron Georg Niebuhr y Leopold von Ranke, fue la base del Historicismo y su perspectiva de que los acontecimientos pasados eran únicos e irrepetibles y su comprensión derivaba de la consideración de los propios contextos históricos. El interés de estos historiadores decimonónicos estuvo centrado entonces en el relato y los acontecimientos políticos y militares, fomentando una historia política al servicio de los poderes legitimados de los Estados Nacionales, J. Casanova Julián, La historia social y los historiadores. ¿Cenicienta o princesa?, Barcelona, 2003, 45-49. En este escenario historiográfico irrumpieron, hacia mediados del siglo XIX, las ideas de Karl Marx y su "filosofía materialista de implantación política y vocación revolucionaria”, Moradiellos, El oficio del historiador... op. cit., 39, proyectando una nueva forma de concebir los tiempos pretéritos. La concepción materialista de la historia pondera las condiciones de existencia en la determinación de la conciencia, aspirando a explicar el proceso de producción material y las relaciones sociales que estructuran al hombre en sociedad. Las causas fundamentales del cambio histórico están en las tensiones existentes en el seno de las estructuras sociales y económicas.

10. J. Huizinga, El otoño de la Edad Media. Estudios sobre la forma de la vida y del espíritu durante los siglos XIV y XV en Francia y en los Países Bajos, Madrid, 1994 [1919]. 
centista, al ser el resultado de la interacción entre los elementos de los cinco sentidos (colores, aromas, sonidos, gustos y texturas) y la búsqueda de un ideal de belleza ${ }^{11}$.

Los antecedentes citados advirtieron sobre un campo inexplorado, difícil de asir y que proporcionaba argumentos para expresar la insatisfacción de los investigadores con la materia general del trabajo del historiador. Una respuesta a esta situación provino de la reacción que protagonizaron los historiadores franceses reunidos en torno a la publicación de Annales. Sus fundadores, Marc Bloch y Lucien Febvre, se propusieron ofrecer una alternativa a la práctica historiográfica dominante, ampliando el campo de trabajo, presentando nuevos temas de indagación y ensayando la aplicación de métodos aportados por otras disciplinas sociales ${ }^{12}$.

Lucien Febvre fue el primero que se interesó, de forma categórica, por la dimensión sensible de la existencia humana. En efecto, participó activamente en el desarrollo de una concepción de la historia que estaría luego en el origen de los estudios sensoriales. Febvre logró colocar a los sentimientos (sensibilidad) y a las emociones en la escena historiográfica de su tiempo en un artículo publicado en el año 1941 titulado La sensibilité et l'histoire: Comment reconstituer la vie affective d’autrefois? Allí identificó los dispositivos que operaban en la generación de las relaciones interpersonales. En dicho artículo, concibió a las emociones como un sistema de incentivos interindividuales que se diversificaban a través de determinadas situaciones y circunstancias, modificando al mismo tiempo las reacciones y la sensibilidad de los individuos con los que interactuaban. Febvre reveló la multiplicidad de definiciones de sensibilidad y de emoción que podían considerarse y apeló a la psicología histórica para abordar el problema que suponía explicar esas variaciones. Para el autor, el lenguaje escrito y oral permitía establecer que las emociones no eran meras reacciones automáticas del cuerpo a las tensiones del mundo exterior, sino que involucraban complejos procesos cognoscitivos y culturales ${ }^{13}$.

Ante los cuestionamientos sobre la legitimidad de este nuevo objeto de estudio, Febvre argumentó que éste ya se incluía en la narrativa histórica pero de forma inconsciente y anacrónica, imponiendo el historiador registros emocionales de su tiempo a épocas anteriores. Al respecto, declaró que «la verdad es que pretender reconstruir la vida afectiva de una época determinada es a la vez una tarea extremadamente atractiva y extremadamente difícil [que] el historiador no tiene el derecho de abandonar» ${ }^{14}$. De esta forma, las emociones fueron concebidas como construcciones culturales. Febvre participó así en la construcción de la Histo-

11. Para un análisis amplio de la veta sensitiva de este historiador, véase F. Ankersmit, "Huizinga on Historical Experience", en D. Howes (ed.), Senses and Sensation. Critical and Primary Sources, Vol. 2: History and Sociology, London, 2018, 23-38.

12. C. A. Aguirre Rojas, La "Escuela" de los Annales. Ayer, Hoy, Mañana, México, 2005.

13. L. Febvre, "La sensibilité et l'histoire: Comment reconstituer la vie affective d'autrefois?", Annales d'histoire sociale, 3, 1-2, 1941, 7.

14. «Le vrai, c'est que, prétendre reconstituer la vie affective d'une époque donnée, c'est une tâche á la fois extrêmement séduisante et affreusement difficile [quoi] l'historien n’a pas le droit de déserter», Febvre, "La sensibilité et l'histoire...", op. cit., 12. Traducción propia. 
ria de las emociones, perspectiva interesada en el estudio de las experiencias afectivas y emocionales del pasado ${ }^{15}$ que contribuiría, de manera decisiva, a la historización de los sentidos ${ }^{16}$.

Las contribuciones de Huizinga y Febvre reconocieron la trascendencia de la materia sensible para el conocimiento de la historia. Este posicionamiento puede ser atribuido, en la medida en que 'toda historia es historia contemporánea' según Benedetto $\mathrm{Croce}^{17}$, al contexto histórico en el que vivieron estos investigadores. De alguna manera se vieron compelidos a pensar en la naturaleza de las motivaciones humanas y en las relaciones sociales en plena transformación, en una generación que conoció y sufrió en carne propia las consecuencias de los conflictos bélicos y el trauma de los totalitarismos.

En definitiva, las primeras décadas del siglo XX fueron decisivas para la creación de las condiciones epistemológicas que hicieron posible el surgimiento de una historia de los sentidos en ciernes y que se consolidaría varias décadas más tarde. Este hecho no nos debe impulsar a pensar en que la materia sensible fue ignorada por el resto de los historiadores sino que, por el contrario, fue tratada en el concierto de inquietudes más generales asociadas, por ejemplo, a las mentalidades. Recién en la segunda mitad del siglo XX, se proyectó estudiar de forma específica la interacción de los sentidos entre sí y la intervención del universo sensorial en la construcción de una cultura particular y las posibilidades que brinda para interpretarla.

En los años sesenta y setenta del siglo pasado, se llevaron a cabo numerosos estudios antropológicos que constituyeron los fundamentos teórico-metodológicos de la Historia de los sentidos ${ }^{18}$ al desnaturalizar la idea de que los sentidos sólo se constituyen como respuesta

15. Esta perspectiva historiográfica ha tenido un gran avance en la segunda mitad del siglo XX, inaugurando un campo de estudio interesante que se nutre de diversas disciplinas sociales. Para estudios actuales véanse los trabajos de J. Plamper, "Historia de las emociones: caminos y retos", Cuadernos de Historia Contemporánea, 36, 2014, 17-29; J. Moscoso, “La historia de las emociones, ¿ de qué es historia?”, Revista Vínculos de Historia, 4, 2015, 28-40; M. Bjerg, "Una genealogía de la historia de las emociones”, Quinto Sol, 23, 1, 2018, 1-20; R. Boddice, The History of Emotions, Manchester, 2018. Una obra de referencia imprescindible es la publicada por Barbara Rosenwein y Riccardo Cristiani donde exponen de forma clara y concisa los enfoques, debates y tendencias pasadas y actuales en la Historia de las emociones y sus principales iniciativas actuales en el campo emocional, B. Rosenwein y R. Cristiani, What is the History of Emotions? (What Is History?), Cambridge, 2018. A comienzo de 2019, se publicó la colección A Cultural History of the Emotions editada por Bloomsbury Academic y bajo la dirección de Susan Broomhall, Jane W. Davidson y Andrew Lynch, cuyos objetivos son explorar cómo las emociones han cambiado a lo largo de la historia, como así también cómo han creado y cambiado la historia. A lo largo de sus seis volúmenes (cada uno dedicado a una época histórica, desde la Antigüedad hasta la actualidad) se manifiesta cómo las emociones pueden ofrecer una visión única del pensamiento histórico. Para el caso medieval, se destacan las obras de B. H. Rosenwein, Emotional Communities in the Early Middle Ages, Ithaca, Cornell University Press, 2006; D. Bouquet y P. Nagy, Sensible Moyen Âge. Une histoire des émotions dans l'Occident médiéval, Paris, 2015.

16. A. Corbin, "Charting the Cultural History of the Senses", en D. Howes (Ed.), Empire of the Senses. The Sensual Culture Reader, Oxford-New York, 2005, 128.

17. R. G., Collingwood, Idea de la historia, México, 1996, 198.

18. Los investigadores de esta corriente han polemizado sobre las categorías de "Historia de los sentidos" e "Historia sensorial" que utiliza la comunidad académica. Para Mark Smith, ambas acepciones no involu-

Revista de historiografía 34, 2020, pp. 277-298 
fisiológica del medio ${ }^{19}$. En efecto, plantearon que estos surgen ante todo «de una orientación cultural que deja margen a la sensibilidad individual $»^{20}$. La antropología proporcionó al historiador una forma para abordar las relaciones de los seres humanos en las múltiples sociedades, con el hecho de ver, oler, gustar, oír y tocar. Para lograr aprehender el funcionamiento de las relaciones sensoriales al interior de las dinámicas sociales, el antropólogo «deconstruye la evidencia social de sus propios sentidos y se abre a otras culturas sensoriales, a otras maneras de sentir el mundo $»^{21}$, observando la manera en que varía la configuración de la experiencia sensorial entre las distintas culturas, según el significado relacionado con cada uno de los sentidos y la importancia que se le confiere. De esta manera, analiza la función de los olores, los gustos, las percepciones visuales, táctiles y auditivas como claves esenciales para entender la manera en que una sociedad crea y plasma un mundo con sentido.

En la década de los ochenta, las percepciones sensoriales se convirtieron en un objeto de estudio delimitado para la historia y la antropología ${ }^{22}$, gracias a los intercambios de conceptos, propuestas y marcos teóricos compartidos entre estas dos disciplinas. El historiador francés Alain Corbin fue el primero en plantear, de forma consciente y sistemática, un análisis sensorial del pasado procurando aprehender la experiencia olfativa y comprender las representaciones que constituyen el imaginario social, así como también las variaciones y desplazamientos de sentido atribuidos a lo sensorial.

Este propósito lo concretó en su obra titulada Le miasme et la jonquille: Lodorat et l'imaginaire social, XVIIIe-XIXe siècles ${ }^{23}$, donde trazó una historia de la percepción olfativa, analizando los modos de percepción, las sensibilidades, el simbolismo de los olores y las prácticas higiénicas en Francia durante el XIX. Aspectos que tienen, según el autor, una historicidad y que intermediaron en la formación de las estructuras sociales y en el control sobre

cran iguales significados. M. Smith, "Producing sense, consuming sense, making sense: perils and prospects for Sensory History”, Journal of Social History, 40, 2007, 841-858. La Historia de los sentidos se aproxima a los sentidos en forma individual explorando sus características principales y desarrollando un camino hacia una historia sensorial. Esta última, por el contrario, tiene una visión ecuménica, buscando la intervención de los sentidos en su conjunto en la construcción social y cultural en el pasado. Se ocupa de dilucidar la forma en que la gente pensaba a los sentidos y el procesamiento cognitivo de sus percepciones sensoriales teniendo en cuenta el contexto social y cultural.

19. Al respecto, se pueden citar los trabajos de M. McLuhan, The Gutenberg Galaxy, Toronto, 1964; W. Ong, "World as View and World as Event", American Anthropologist, 71, 1969, 634-647, W. Ong, Orality and Literacy, New York, 1982; E. Carpenter, Oh, What a Blow that Phantom Gave Me!, Toronto, 1972; C. LéviStrauss, The Savage Mind, Chicago, 1966; C. Lévi-Strauss, The Raw and the Cooked: Introduction to a Science of Mythology, vol. 1, New York, 1969.

20. D. Le Breton, El sabor del mundo. Una antropología de los sentidos, Buenos Aires, 2007, 13.

21. Le Breton, El sabor del mundo..., op. cit., 16.

22. Para un análisis de las tres genealogías sensoriales dentro de esta ciencia, véase T. Porcello, L. Meintjes, A. M. Ochoa, and D. W. Samuels, "The Reorganization of the Sensory World", Annual Review of Anthropo$\log y, 39,2010,51-66$.

23. A. Corbin, El perfume o el miasma. El olfato y lo imaginario social, siglo XVIII y XIX, México, 1987 [1982]. 
los cuerpos ${ }^{24}$. Para Corbin, «La historia social, respetuosa de los humildes, pero durante muy largo tiempo sorda a la expresión de sus afectos, no debe ya callar las reacciones primarias, así éstas fuesen sórdidas, con el pretexto de que la antropología delirante de la era darviniana había pervertido el análisis» ${ }^{25}$. Con ello, el autor exhortó a los historiadores a examinar a las percepciones en relación al modo en que intervienen en la disposición de las jerarquías sociales, tal como observó en la sociedad francesa donde los olores determinaron la posición social de los sujetos en el imaginario colectivo.

Distintos investigadores se hicieron eco de este desafío, pero en nuestra opinión, fueron dos de ellos en particular los que marcaron el camino de los estudios sensoriales: C. Classen y D. Howes. Sus trabajos se convirtieron en la materia prima indispensable de la reflexión teórica y metodológica sobre el tema, proporcionando herramientas imprescindibles para cualquier historiador que tenga intenciones de iniciarse en este campo.

Constance Classen fue una de las primeras estudiosas en plantear la discusión sobre la dimensión intercultural de algunos de los aspectos sociales de la percepción y las prácticas sensoriales. En sus primeros trabajos, expuso cómo el olor se convirtió en un marcador de identidad social y cultural en la sociedad norteamericana al analizar el modo en que se transformó en un dispositivo que empleaban los grupos dominantes (blancos) para afirmar su posición frente a la población subalterna ${ }^{26}$. El interés de la autora por el sentido del olfato continuó en otras dos publicaciones en las que abordó las distintas significaciones atribuidas a los olores en diversas configuraciones sociales ${ }^{27}$. Asimismo, avanzó en el examen de las prácticas y las percepciones táctiles y puso especial atención en descifrar cómo sus significaciones mutaron en la transición de una cultura premoderna hacia una cultura moderna del sentido visual $^{28}$. En relación con ello, en su trabajo The Color of Angels: Cosmology, Gender and the Aesthetic Imagination ${ }^{29}$, Classen indagó sobre la relación de los sentidos (principalmente la vista y los colores) y las visiones religiosas sensuales desde la Edad Media hasta el siglo XIX.

El desafío de todo análisis sensorial está en superar la mera identificación y descripción de las percepciones. El resultado final al que debería aspirar este abordaje es un análisis holístico de las relaciones que se establecen entre los sentidos, en pos de la explicación de su intervención en la aprehensión del mundo por parte de los sujetos. Este reto

24. Este proceso disciplinario fue abordado por Norbert Elias en la década de los años treinta del siglo XX al analizar la evolución de las costumbres y de los comportamientos a partir del renacimiento. A través de ello, propuso una visión de conjunto de los cambios sociales y culturales que se produjeron en la sociedad cortesana, desarrollando una teoría del proceso de civilización y la sociología figurativa, N. Elias, La sociedad cortesana, México, 1996 [1969]; N. Elias, El proceso de civilización, Madrid, 2011 [1939].

25. Corbin, El perfume o el miasma..., op. cit., 249.

26. C. Classen, "The Odor of the Other: Olfactory Symbolism and Cultural Categories", Ethos, 20, 2, 1992, $133-166$.

27. C. Classen, Worlds of Sense... op. cit; C. Classen, D. Howes y A. Synnott, Aroma: The cultural history of smell, New York, 1994.

28. C. Classen, The Book of Touch, Oxford, Berg, 2005; C. Classen, The Deepest Sense: A Cultural History of Touch, Champaign, 2012.

29. C. Classen, The Color of Angels: Cosmology, Gender and the Aesthetic Imagination, London, 1998. 
fue asumido por Classen en su obra Inca Cosmology and the Human Body ${ }^{30}$, donde analizó la forma en que la sociedad incaica generó sentidos sobre el mundo en que vivía por medio de metáforas y prácticas corporales y sensoriales en los tiempos de la conquista española. El andamiaje teórico y metodológico necesario para ello fue construido por la autora a través de la exploración de una serie de modelos y prácticas basadas en las significaciones que los sentidos poseían en disímiles culturas.

Gracias a estos trabajos, Classen formuló una crítica que la distanció de los trabajos científicos que le precedían y trazó el camino para una nueva forma de emprender el estudio de los sentidos. En efecto, consideró que era necesario establecer la vinculación de todos los sentidos que una cultura define y experimenta más allá de la importancia decisiva otorgada a cada uno de ellos frente al resto. De esta manera, estimuló el estudio global de los sentidos y la interrelación que se establece entre éstos, los individuos y la sociedad destacando que cualquier escisión que pudiera realizar el investigador era meramente analítica.

Por su parte, David Howes comenzó su incursión en el campo de la Historia de los sentidos analizando las metáforas gustativas y olfativas utilizadas por los intelectuales europeos del siglo XVIII para dar cuenta de las tensiones y las diferencias sociales de su época ${ }^{31}$. En sus investigaciones, Howes propuso un examen global de los sentidos y desarrolló los parámetros y criterios que, en su opinión, debían seguirse para aplicar un método comparativo con el objetivo de destacar los contrastes entre los órdenes sensibles de las distintas culturas ${ }^{32}$.

La particularidad de estas pesquisas es el trabajo interdisciplinario que llevan a cabo sus autores, no sólo como un anhelo o una meta posible, sino como resultado tangible que se expone en publicaciones colectivas que recogen distintas corrientes historiográficas y categorías analíticas. Una de las primeras iniciativas editoriales que demuestra este proyecto estuvo a cargo de Constance Classen, David Howes y Anthony Synnott ${ }^{33}$. Se trató de la primera exploración sobre la función cultural de los olores en diferentes períodos de la historia de Occidente y una amplia gama de sociedades no occidentales. A lo largo de sus páginas, las contribuciones plantearon el análisis de una gran variedad de fuentes y, en consecuencia, una explicación histórica y transcultural de las creencias y prácticas en materia del olfato. Este libro atestiguó que toda sensación se constituye en un elemento cultural por el cual se difunden los valores y prácticas de una sociedad y se estructuran los roles sociales que desempeñan los sujetos ${ }^{34}$.

30. C. Classen, Inca Cosmology and the Human Bod, Salt Lake City, 1993.

31. D. Howes y M. Lalonde, "The history of sensibilities: of the standard of taste in mid-eighteenth century England and the circulation of smells in post-revolutionary France", Dialectical Anthropology, 16, 1991, 125-135.

32. D. Howes, The Varieties of Sensory Experience, Toronto, 1991; D. Howes, Sensual Relations: Engaging the Senses in Culture and Social Theory, Michigan, 2003.

33. C. Classen, D. Howes y A. Synnott, Aroma..., op. cit., 1994.

34. Un rasgo que distingue a esta obra es la atención que presta al cuerpo en relación con los sentidos. La cuestión se planteó por medio de los interrogantes de cómo y por qué se construye el cuerpo y, a su vez, por qué estas construcciones varían y cambian a lo largo del tiempo. El cuerpo no es "dado", sino una categoría 
La segunda obra que marcó un hito en el campo de los estudios sensoriales, fue Empire of the senses: the sensual culture reader ${ }^{35}$. A lo largo de sus capitulos se brinda, desde diversas disciplinas (Antropología, Historia, Etnomusicología, Filosofía, Sociología, Geografía, Museología, Derecho, Neurología y Literatura), un análisis cultural de la experiencia sensorial, concibiendo a los sentidos como medios de acceso a las ideas que permiten conocer la forma en que operan los instrumentos de poder y las fuentes de placer y dolor de sociedades pasadas y presentes ${ }^{36}$.

El recorrido historiográfico realizado permitió puntualizar los problemas específicos de la Historia de los sentidos. Quedan por mencionar algunos de los desafíos y limitaciones que afronta la investigación sensorial. Al respecto, Corbin alertó que una parte considerable de la documentación a la que se puede recurrir brinda datos escasos y fragmentarios, difíciles de ponderar según su naturaleza ${ }^{37}$. En sus palabras: «El obstáculo más obvio reside en la fugacidad de la huella» ${ }^{38}$. El historiador debe ser capaz de descifrar aquellas referencias sensoriales disponibles y detectar la lógica que subyace en ellas en función de las convenciones científicas dominantes en el periodo estudiado. A pesar de ello, sostiene que esta «historia en fragmentos», que se trasmite bajo múltiples mediaciones, permite comprender retrospectivamente el pasado a través del análisis de la jerarquía de los sentidos y valida la existencia de una historia de la sensorialidad.

En los últimos años, con estos preceptos en mente, los medievalistas comenzaron a ser conscientes de la naturaleza cultural de la materia sensitiva y emprendieron una indagación específica para el medioevo. A continuación, trazaremos el curso de las investigaciones sobre esta temática para la época medieval, destacando sus particularidades y disparidades regionales en la exploración, y proyectos de interés en curso.

\section{La Edad Media a través de los sentidos: iniciativas historiográficas y desafíos}

El abordaje sensorial de los tiempos medievales de Occidente ${ }^{39}$ es una empresa reciente a causa de la complejidad que presentan las fuentes disponibles para su análisis. No obstante,

social con diferentes significados impuestos y desarrollados por todas las edades y diferentes sectores de la población. Por lo tanto, se asemeja a una "esponja" puesto que posee una capacidad para absorber distintos significados (sociales, culturales y políticos) C. Classen, D. Howes y A. Synnott, Aroma... op. cit., 1994.

35. D. Howes (ed.), Empire of the senses: the sensual culture reader, Oxford-New York, 2005.

36. C. Classen y D. Howes, Ways of Sensing: Understanding the Senses In Society, New York, 2013.

37. A. Corbin, "Histoire et anthropologie sensorielle", Anthropologie et Sociétés, 14, 2, 1990, 17.

38. «Lobstacle le plus évident réside dans la fugacité de la trace», Corbin, "Histoire et anthropologie sensorielle”..., op. cit., 15. Traducción propia.

39. Para el caso Oriental se pueden citar las obras de L. James, "Senses and sensibility in Byzantium", en Art History, 27, 4, 2004, 522-537; S. Antonopoulos et al., "Soundscapes of Byzantium", Speculum, 92, 2017, 321-335; R. Betancourt, Sight, touch, and imagination in Byzantium, Cambridge, 2018. 
se pueden destacar, al menos, dos razones por lo que resulta sugestivo emprenderla. En primer lugar, por la alteridad y la ambigüedad de la información sensorial transmitida por los textos medievales y, en segundo lugar, por el descrédito que sufrieron los sentidos en muchas de las obras teológicas que resulta necesario revertir ${ }^{40}$.

Éric Palazzo, uno de los especialistas contemporáneos más reconocidos en este campo, ofreció un recorrido bibliográfico sobre las iniciativas interesadas en los sentidos en la época medieval desde la segunda mitad del siglo XX, destacando los análisis históricos, literarios, filosóficos, antropológicos, sociológicos, artísticos y religiosos existentes ${ }^{41}$. Este autor subraya la importancia de utilizar la noción de synesthésie para dar cuenta del efecto sensorial producido por la interacción entre los cinco sentidos, concepción que se asemeja a la intersensoriality ${ }^{42}$ planteada por los investigadores anglosajones. Para Palazzo, esta última noción remite a una fase anterior a la sinestesia anhelada.

La curiosidad por los sentidos medievales comenzó a vislumbrase desde finales de los años setenta del siglo XX en el área de los estudios sobre el arte y la estética, observando la potencialidad del análisis de las percepciones sensoriales y las expresiones artísticas. Carl Nordenfalk fue uno de los primeros en plantear la relación existente entre determinados sentidos y la representación pictórica de animales, pecados capitales, órganos y grupos sociales en murales, pinturas y textos de la Edad Media y el Renacimiento ${ }^{43}$. En esta misma línea, François Quiviger examinó la relación entre el arte y los sentidos en el Renacimiento como representación de una cultura sensorial concebida, en términos generales, como la suma de experiencias, hábitos y supuestos necesarios para generar una aprehensión y empatía con las imágenes. La tradición alegórica medieval es el punto de acceso a la función de los sentidos y las sensaciones en las artes visuales, al estudiar el arte religioso y el laico ${ }^{44}$.

El segundo enfoque se centra en el campo de la literatura, la liturgia, los manuales de confesión y los penitenciales medievales. Palazzo prestó atención a la íntima relación que se establece entre los sentidos, la liturgia y las imágenes ${ }^{45}$. Para este autor, la comprensión del

40. R. Newhauser, “Theory and Practice: The Senses in the Middle Ages”, Senses \& Society, 4, 3, $2009,367$.

41. E. Palazzo, "Les cinq sens au Moyen Âge: état de la question et perspectives de recherche", en Cahiers de civilisation médiévale, 4, 55, 2012, 339-366. A modo de ejemplo, véase P. Dronke, "Les cinq sens chez Bernard Silvestre et Alain de Lille", Micrologus, 10, 2002, 1-14; M. Pastoureau, "Le Bestiaire des cinq sens (XIIe-XVIe siècle)", Micrologus, 10, 2002, 133-145.

42. M. Smith, Sensing the Past: Seeing, Hearing, Smelling, Tasting, and Touching in History, Berkeley, $2007,8$.

43. C. Nordenfalk, "Les Cinq Sens dans l'art du Moyen-age", Revue de l'art, 34, 1976, 17-28; C. Nordenfalk, "A unique five- senses cycle of the 1620's", en Journal of Art History, 59, 3, 1984, 183-189; C. Nordenfalk, "The Five Senses in Late Medieval and Renaissance Art", Journal of the Warburg and Courtauld Institutes, 48, 1985, 1-22.

44. F. Quiviger, The Sensory World of Italian Renaissance Art, London, 2010; F. Quiviger, "Art and the Senses: Representation and Reception of Renaissance Sensations", en H. Roodenburg Ed.), A Cultural History of the Senses in the Renaissance, New York- London, 2014, 169-202.

45. Cécile Voyer resaltó la importancia de la dimensión sensorial o el entorno sensorial de las obras visuales medievales, principalmente religiosas. Para esta autora, en los últimos años los análisis de algunos medievalistas sobre las relaciones entre las imágenes y el espectador se han nutrido de las propuestas de los 
papel de los cinco sentidos en la cultura medieval es esencial para precisar algunos aspectos fundamentales del culto y de la teología cristiana que permitirían una mejor interpretación del ritual medieval ${ }^{46}$. La base del mundo sensorial cristiano estuvo determinada por la tradición bíblica y el pensamiento de los Padres de la Iglesia al precisar que el diálogo establecido entre el hombre y la divinidad se realizaba por medio del cuerpo y los sentidos. La indagación llevada a cabo por Palazzo sobre la relación entre el arte, la liturgia y la dimensión sensorial se realizó a través del análisis de las ilustraciones de los libros litúrgicos. La imagen, en su opinión, fue el vehículo por el cual se activaban los sentidos en su conjunto durante el ritual, con el fin de producir el efecto teológico necesario en el ceremonial litúrgico. En consecuencia, para este autor la liturgia se convertía en un «área de sensibilidad», donde la sinestesia creaba las condiciones para la manifestación de lo invisible a través del contenido visible de la experiencia sensorial, elemento esencial de la actuación litúrgica ${ }^{47}$.

Este aspecto de la religión cristiana es uno de los elementos que más fuerza tiene dentro de los análisis sensoriales medievales. La relación del cristianismo con los sentidos es analizada en la obra dirigida por Paul Gavrilyuk y Sarah Coakley ${ }^{48}$, cuyo objetivo primordial fue explicar cómo y por qué los escritores cristianos (patrísticos y teólogos) emplearon una serie de términos e imágenes sensoriales para articular el encuentro del hombre con la divinidad.

En otro orden de relaciones, el lugar que ocuparon las percepciones en la experiencia religiosa y en la vida doméstica fue el interrogante que se planteó Christopher M. Woolgar. El autor analizó este tópico en la sociedad inglesa de la Baja Edad Media ${ }^{49}$, destacando el significado moral que poseían los cinco sentidos en los manuales de confesión y la cultura material. Además de desarrollar las teorías sobre los sentidos y su interpretación por parte de la sociedad medieval, Woolgar intentó dilucidar cómo éstos se aplicaron en la práctica en contextos predominantemente domésticos de la nobleza ${ }^{50}$. A lo largo de la obra, se interrogó sobre la relación íntima que existía entre el pecado y la virtud de los sentidos y la forma en que éstos se interpretaron en la vida cotidiana.

En esta misma línea de análisis, Carla Casagrande observó los vínculos entre los senti$\operatorname{dos}^{51}$ y la clasificación de los pecados en los penitenciales de los siglos XII y XIII, destacando

estudios sensoriales, gracias a los cuales han planteado el papel fundamental que cumplía de una representación u objeto en la activación de los sentidos durante los ritos litúrgicos. C. Voyer, "Les cinq sens et les images au Moyen Âge: voir et revoir les œuvres visuelles médiévales”, Quaestiones Medii Aevi Novae, 21, 2017, 227-249.

46. E. Palazzo, L'invention chrétienne des cinq sens dans la liturgie et l'art au Moyen Âge, Paris, 2014.

47. E. Palazzo, "Art, liturgy, and the five senses in the Early Middle Ages", Viator, 41, 1, 2014, 25-56.

48. P. Gavrilyuk y S. Coakley (Ed.), The Spiritual Senses: Perceiving God in Western Christianity, Cambridge, 2012.

49. Para un estudio de la relación de los sentidos en el proceso posterior de la reforma en Inglaterra puede verse la obra de M. Milner, The Senses y the English Reformation, Burlington, 2011.

50. C. Woolgar, The Senses in Late Medieval Engly, New Haven, 2006.

51. En un abordaje previo, Carla Casagrande y Silvana Vecchio estudiaron el pecado de scurrilitas (las burlas o palabras vanas e inútiles que provocan) contra el que clamaban los predicadores y los moralistas de 
que las percepciones sensoriales fueron objeto del discurso moral de la Iglesia transmitido a la comunidad de fieles ${ }^{52}$. Cada sentido se identificó con un pecado en particular, estableciéndose una jerarquía entre ellos. En opinión de la autora, los sentidos poseían una doble naturaleza. Por un lado eran instrumento de la perdición y pecado y, por el otro, participaban de la salvación al ser las puertas de entrada de la gracia divina ${ }^{53}$.

El mundo sensorial de la cultura cristiana medieval ${ }^{54}$ fue el objeto de estudio de la obra coordinada por Annette Kern-Stähler, Beatrix Busse y Wietse de Boer, titulada The Five Senses in Medieval and Early Modern England ${ }^{55}$. A lo largo de sus páginas se examinó las interrelaciones entre las percepciones y la cultura (secular y cristiana) de Inglaterra desde la Edad Media hasta los tiempos temprano modernos, a través de los textos canónicos, la poesía, el teatro, la homilétia, el martirologio y los escritos eruditos de la época. Este material fue abordado por medio de un marco teórico metodológico integrado por los campos de la lingüística del cuerpo, los estudios de traducción, la historia del arte y la arqueología, junto con los enfoques originarios de los estudios literarios tradicionales. Los autores realizaron una exploración de las funciones desempeñadas por los sentidos en la literatura, la liturgia y el teatro, cuyas representaciones sensoriales participaron en la construcción social de diversas categorías, tales como el pecado, la salvación, la ilusión, la realidad y el binomio «yo-mundo».

Este abordaje de la época medieval se expandió gracias al trabajo interdisciplinario entre historiadores y estudiosos de la literatura de las lenguas romances ${ }^{56}$. En esta perspectiva, la obra Rethinking the Medieval Senses: Heritage / Fascinations / Frames ${ }^{57}$, nos aproxima a la sensorialidad medieval desde cuatro vertientes. En primer lugar, desarrolló las conceptualizaciones filosóficas en la obra de Agustín de Hipona y exploró el juicio sobre la fisiología de la sensación y la función del sentido interno como mecanismo de control de la percepción. En segundo lugar, indagó sobre las representaciones literarias de los cinco sentidos, deteniéndose en la «sensación bucal» de la risa, y en el reflejo de comer, beber y hablar, en obras his-

la Edad Media, C. Casagrande y S. Vecchio, I peccati della lingua. Disciplina ed etica della parole nella cultura medievale, Roma, 1987.

52. El análisis de las percepciones sensoriales, en algunos contextos, se desarrolla en consonancia con las emociones, puesto que ambos procesos se desarrollan en forma simultánea, como demuestra Casagrande en un estudio donde se detiene en la dimensión emocional de los sacramentos. C. Casagrande (2012), "Le emozioni e il sacramento della penitenza”, en M. Sodi y R. Salvarani (Coord.), La penitenza tra I e II millennio. Per una comprensione delle origini della Penitenzieria Apostoloca, Cittá del Vaticano, 2012, 213-231.

53. C. Casagrande, "Sistema dei sensi e classificazione dei peccati (secoli XII-XIII)", Micrologus, 10, 2002, 33-54.

54. La importancia de la percepción sensorial en la devoción medieval fue analizada por B. Williamson, "Sensory Experience in Medieval Devotion: Sound y Vision, Invisibility y Silence", Speculum, 88, 1, 2013, 1-43.

55. A. Kern-Stähler, B. Busse y W. de Boer, The Five Senses in Medieval y Early Modern Engly, LeidenBoston, 2016.

56. En esta misma línea, la obra Sensory Perception in the Medieval West se interroga sobre la percepción sensorial desde un punto de vista literario y material: S. Thomson y M. Bintley (Ed.), Sensory Perception in the Medieval West, Utrecht Studies in Medieval Literacy, Turnhout, 2016.

57. S. Nichols, Y. Kablitz y A. Calhoun (Ed.), Rethinking the Medieval Senses: Heritage / Fascinations / Frames, Baltimore, 2008. 
pánicas e italianas de fines de la Edad Media. En tercer lugar, analizó los subtextos culturales de «lo sensorial» en la ética y la política. Por último, interrogó a los sentidos desde la óptica de la fisiología y la filosofía. En su conjunto, la obra expuso una comprensión teórica de los sentidos y su importancia en la historia intelectual medieval.

Otro hito historiográfico importante para la Historia de los sentidos fue la publicación, en el año 2014, de la colección A Cultural History of the Senses coordinada por Constance Classen, cuyos seis volúmenes introducen al lector en las bases sensoriales de la civilizació n occidental, desde la Antigüedad hasta la Edad Contemporánea, por medio de un enfoque integral ${ }^{58}$. Los temas que se abordaron, ejes vertebradores de la obra, fueron los siguientes: los marcadores sensoriales de género y de clase, las dimensiones estéticas de la cultura material y las sensibilidades religiosas, los usos médicos de los sentidos y su representación en el arte y la literatura, entre otros. Todos los contenidos se estructuraron en capítulos específicos ${ }^{59}$ que se retomaron en cada volumen con el objetivo de permitir una lectura transversal de todas las etapas históricas. A lo largo de sus páginas, el lector puede constatar la factibilidad de considerar a los sentidos como objetos de estudio para el conocimiento de una sociedad en su dimensión sensorial.

El tercer enfoque centra su atención en la intervención de los sentidos en la vida cotidiana y en los espacios urbanos. Esta inquietud se materializó en el libro titulado Les cinq sens de la ville, coordinado por Robert Beck, Ulrike Krampl y Emmanuelle Retaillaud-Bajac ${ }^{60}$. Esta obra ofrece una exposición de las distintas perspectivas históricas y antropológicas de los paisajes sensoriales de la Edad Media hasta nuestros días, señalando la experiencia íntima y social - concebida por las expectativas culturales y emocionales- que poseen los espacios urbanos. La ciudad era comprendida en una doble perspectiva, tanto en su materialidad como su inagotable fuente de estimulación, al ser proporcionada por la experiencia de los habitantes e informada por la memoria de sus cuerpos. Esta obra buscó problematizar la relación entre el espacio y los sentidos al considerarlos los resortes principales de la construcción de la ciudad como un todo significante a lo largo del tiempo ${ }^{61}$.

58. J. Toner (Ed.), A Cultural History of the Senses in Antiquity, 500 BCE-500 CE, New York-London, 2014; R. Newhauser (Ed.), A Cultural History of the Senses in the Middle Ages, 500-1450, New York-London, 2014; H. Roodenburg (Ed.), A Cultural History of the Senses in the Renaissance, 1450-1650, New YorkLondon, 2014; A. Vila (Ed.), A Cultural History of the Senses in the Age of Enlightenment, 1650-1800, New York-London, 2014; C. Classen (Ed.), A Cultural History of the Senses in the Age of Empire, 1800-1920, New York-London, 2014; D. Howes (Ed.), A Cultural History of the Senses in the Modern Age, 1920-2000, New York-London, 2014.

59. The Social Life of the Senses; Urban Sensations; The Senses in the Marketplace; The Senses in Religion; The Senses in Philosophy y Science; Medicine y the Senses; The Senses in Literature; Art y the Senses; y Sensory Media.

60. R. Beck, U. Krampl y E. Retarllau- Bajac (Dirs.), Les cinq sens de la ville, Tours, 2013.

61. Desde esta perspectiva desarrollamos nuestra tesis doctoral titulada "La construcción social del paisaje sonoro de las ciudades castellanas (c. 1400 - c. 1560)", cuyo principal objetivo fue analizar y construir el paisaje sonoro de los núcleos urbanos del Reino de Castilla entre los años c. 1400 y c. 1560, objeto escasa- 
En los últimos años, los estudios sensoriales medievales comenzaron a indagar sobre la relación entre los objetos materiales y la experiencia, aspecto que John H. Arnold destacó al declarar que los enfoques centrados en los sentidos y en las interacciones entre las personas y los objetos materiales forman parte de la futura agenda historiográfica ${ }^{62}$. Un ejemplo de esta orientación es la obra coordinada por Fiona Griffiths y Kathryn Starkey Traces of Experience in Medieval Artifacts ${ }^{63}$, que inaugura la colección Sensor Reflections: Sense, Matter, and Medium, cuyo objeto de análisis son los artefactos medievales, su manipulación social y la experiencia sensorial que producen en los sujetos históricos. Este abordaje se realiza desde una reflexión de la experimentación práctica, dejando de lado la teoría sensorial de los eruditos y exégetas medievales. La premisa epistemológica subyacente en la obra sostiene que los objetos, además de relatar una historia diferente a la de los textos, manifiestan particularidades de las comunidades, tanto de su vida cotidiana como de sus elementos culturales y de las personas que no pertenecen a las élites, datos que difícilmente se puedan obtener de la práctica escrituraria habitual ${ }^{64}$. Un punto interesante para destacar es que esta obra se encuentra en la intersección de dos campos en potencia: la cultura material medieval y la experiencia sensorial ${ }^{65}$, prestando atención a las huellas de prácticas efímeras y físicas que rara vez se articularon o describieron en los textos ${ }^{66}$. En consecuencia, esta propuesta plasma la potencialidad de un abordaje sensorial de la Edad Media en sus aspectos materiales y simbólicos, sin desconocer las dificultades metodológicas y documentales que se plantean al momento de emprender este camino.

Hasta aquí expusimos las iniciativas historiográficas que se centraron en el análisis de la totalidad de los sentidos. Sin embargo, los investigadores tienden a privilegiar en sus abordajes a un sentido más que a otro a causa de la relevancia que tiene la jerarquía sensorial en el mundo occidental. Este es el caso del sentido auditivo y los sonidos captados por él. Los medievalistas han intentado interpretar y comprender las relaciones sensoriales de la época por medio del análisis de temas específicos, como la música ${ }^{67}$ sacra y profana, el rumor en la

mente abordado por los estudios dedicados a los últimos siglos medievales. Para una mayor explicación de la misma, véase G. Coronado Schwindt, "Resumen de Tesis: "La construcción social del paisaje sonoro de las ciudades castellanas (c. 1400 - c. 1560)”, Anuario de la Escuela de Historia Virtual, Facultad de Filosofía y Humanidades, Universidad Nacional de Córdoba, 14, 120-126, 2018.

62. J. H. Arnold, "Histories y Historiographies of Medieval Christianity,", en J. H. Arnold (Ed.), The Oxford Handbook of Medieval Christianity, Oxford, 2014, 38.

63. F. Griffiths y K. Starkey (Ed.), Sensory Reflections: Traces of Experience in Medieval Artifacts, Berlin, de Gruyter, 2018.

64. F. Griffiths y K. Starkey, "Sensing Through Objects", en Griffiths y Starkey Sensory Reflections... op. cit., 13.

65. B. Williamson, "Reflections on Sensory Reflections: An Afterword”, en Griffiths y Starkey Sensory Reflections... op. cit., 249.

66. Griffiths y Starkey, “Sensing Through Objects...”, op. cit., 2.

67. La dimensión sonora puede ser analizada por medio de múltiples plataformas, no sólo el soporte escrito. Susan Boynton planteó un análisis de los sonidos, la música y sus instrumentos en la época medieval a través de la iconografía plasmada en los manuscritos y las esculturas. Para esta investigadora, el arte visual 
esfera política, los gritos y los llantos en los ritos funerarios, etc. Los sonidos se constituyeron en un objeto de análisis independiente del resto de los sentidos al abordarse a través de cuestiones tradicionales de la historiografía medieval.

Uno de los primeros intentos de otorgar un lugar preponderante al hecho sonoro en el análisis histórico, en consonancia con la tendencia historiográfica de la época ${ }^{68}$, fue el estudio de Claude Gauvard y Altan Gokalp sobre la función social y el significado del término charivari en la sociedad francesa de la Baja Edad Media ${ }^{69}$. Estos autores destacaron el papel que cumplieron determinados ruidos en las sociedades tradicionales al manifestar la oposición universal de la equivalencia entre los sexos y la del cielo con la tierra. De la misma forma, analizaron la diferenciación entre ruido y silencio, y la participación que tenían estas dimensiones en los rituales y las celebraciones. Para Gauvard y Gokalp, a finales del siglo XIV y principios del XV la cencerrada (charivari) estuvo sujeta a reglas de conducta específicas, mientras que el alboroto fue considerado un rito de paso a través del cual los jóvenes expresaron los problemas generados por su inserción en una sociedad jerarquizada y en trasformación, cuya consecuencia más importante fue el resurgimiento de las organizaciones juveniles que funcionaron como canales de expresión «sonora» de sus reclamos ${ }^{70}$.

La medievalista argentina Nilda Guglielmi se propuso, en la década de los noventa, comprender cómo una obra literaria - en tanto testimonio histórico- expresaba un imaginario particular por medio de determinados colores y sonidos ${ }^{71}$. En su análisis del cantar ruso del siglo XII, «El Cantar de la Gesta de Igor», observó que además de los recursos estéticos empleados, el autor plasmó la importancia del imaginario cromático y auditivo en el relato. Los sonidos del aparato bélico, de la batalla y del dolor de la derrota, fueron claves en la construcción de una dimensión dramática de la narración que dotaron de identidad a los hombres de esa sociedad.

Las cuestiones sonoras en la época medieval comenzaron a ser abordadas mediante la categoría soundscape (paisaje sonoro) proveniente de la musicología. Con este concepto se

también evocó el sonido de forma indirecta a través de la representación del sujeto de escucha, destacando la necesidad y el potencial de los estudios centrados en la representación visual del silencio y del paisaje sonoro, S. Boynton, "The visual representation of music and sound", en C. Hourihane (ed.), The Routledge Companion to Medieval Iconography, New York, 2017, 943-968.

68. En la década de los setenta, el rito de la cencerrada comenzó a tener importancia en los análisis de la cultura popular europea de la Edad Moderna, gracias a los trabajos de Natalie Zemon Davis y Edward Palmer Thompson, entre otros. Véase N. Zemon Davis, "The Reasons of Misrule: Youth Groups and Charivaris in Sixteenth-Century France", Past \& Present, 50, 1971, 41-75, E. P. Thompson, "Rough Music: le charivari anglais", Annales. Économies, Sociétés, Civilisations, 2, 1972, 285-312, J. Le Goff y J. C. Schmitt (ed.), Le charivari, Paris, 1981.

69. C. Gauvard y A. Gokalp, "Les conduites de bruit et leur signification à la fin du Moyen Âge: le charivari”, Annales. Économies, Sociétés, Civilisations, 3, 1974, 693-704.

70. Gauvard y Gokalp, "Les conduites de bruit et leur signification..., op. cit., 698-704.

71. N. Guglielmi, "El imaginario cromático y auditivo en el Cantar de la Gesta de Igor", en R. Rodríguez (dir.), Saber, pensar, escribir, La Plata, 2011 [1992], 213-246. 
identificó al entorno sonoro de un lugar, compuesto por los sonidos del individuo y la naturaleza $^{72}$. La historiografía francesa ha sido precursora en aplicar esta noción a los análisis históricos. Jean-Marie Fritz fue uno de los primeros medievalistas en plantear el estudio del sonido y la audición en la Edad Media mediante una perspectiva epistemológica. La misma permite considerar al sonido como objeto de conocimiento, con el propósito de reconstruir el paisaje sonoro del universo medieval, sin reducirlo a su dimensión visual. Su objetivo primordial fue explorar los distintos ámbitos de la cultura medieval a través de la arqueología, la literatura y la teología de la liturgia, con la intención de proponer una visión global de la sonoridad. El desafío de esta obra fue examinar los tiempos medievales de acuerdo a un punto de vista acústico, centrándose en las imágenes y los textos de las obras musicales y patrísticas ${ }^{73}$, otorgándole un lugar destacado a la voz, tanto en su dimensión teológica como cristológica, y al ruido provocado por los elementos naturales. Para Fritz, la originalidad de la Edad Media consistió en el entendimiento del sonido como fenómeno vocal del mundo, es decir, que todas las manifestaciones - naturales y humanas - se vocalizaron a partir del rastro de la voz suprema de la palabra creadora del Génesis y, espacialmente, abriendo el camino a una interpretación moral y alegórica.

Por su parte, Jean-Pierre Gutton en su obra titulada Bruits et sons dans notre histoire. Essaisuu la reconstitution du paysage sonore, sostuvo que los sonidos y ruidos de una época posibilitan estudiar los entornos de la vida cotidiana, los conflictos y las solidaridades hacia el interior de una sociedad ${ }^{74}$. En el capítulo dedicado a la época medieval, Gutton destacó los sonidos que se producían durante las ruidosas ceremonias reales, donde reyes y príncipes reafirmaban su poder y legitimidad. Las entradas reales eran anunciadas por medio de las trompetas y los tambores con el objetivo de asegurar la transmisión del discurso regio a los súbditos $^{75}$. La ciudad medieval poseía una sonoridad particular que fue nutrida por los sonidos cívicos, artesanales y religiosos que desprendían sus habitantes. Para este autor, la Edad Media se destacó por los ruidos de sus festividades, sus actividades artesanales y los sonidos de los campanarios, enfatizando la búsqueda del silencio en determinados lugares y sujetos, como fueron los espacios sacros (monasterios e iglesias) ${ }^{76}$. Asimismo, a pesar de destacar las manifestaciones sonoras presentes en este periodo histórico, no se detiene en los paisajes sonoros de la época medieval de forma extensa, puesto que considera que la Edad Moderna es la que ostenta los entornos sonoros más complejos.

72. A fines de la década de los años sesenta y principios de los setenta, un grupo de científicos, liderados por Raymond Murray Schafer, crearon el Proyecto Paisaje Sonoro Mundial (WSP) en la Universidad Simón Fraser (Canadá) con el objetivo de llamar la atención sobre el medio ambiente sonoro a través de un curso sobre la contaminación sonora, de allí surge el concepto de paisaje sonoro para analizar las diversas expresiones sonoras actuales, R. M. Schafer, The Tuning of the World, Toronto, 1977.

73. J. M. Fritz, Paysages sonores du Moyen Age: le versant épistémologique, Paris, 2000, 8.

74. J. P. Gutton, Bruits et sons dans notre histoire. Essaisuu la reconstitution du paysage sonore, Paris, 2000, $5-17$.

75. Gutton, Bruits et sons dans notre histoire... op. cit., 19-20.

76. Gutton, Bruits et sons dans notre histoire... op. cit., 20-27. 
En la misma línea de análisis, Laurent Vissière manifestó la composición y complejidad del paisaje sonoro de la ciudad de París durante los siglos XIII al XIV, a través del estudio de obras literarias, musicales e iconográficas ${ }^{77}$. Para Vissière, la clave de la significación de ciertos sonidos en la dinámica ciudadana parisina estuvo en los gritos de quienes anunciaban la venta de alimentos y de diversos objetos ${ }^{78}$. En el año 2016 dirigió, junto con Laurent Hablot, una obra que reunió aportes de distintos investigadores provenientes de la historia, la literatura y la musicología, interesados en las diversas expresiones sonoras producidas por el hombre y su entorno - en tanto música vocal e instrumental como sonidos de la naturaleza, los gritos y las palabras- que conformaron el paisaje sonoro de los territorios de la Borgoña francesa e Italia del periodo comprendido entre los años 1400 y 1550. A lo largo de sus capítulos se indagaron los testimonios sonoros que acompañaron a las instancias de justicia, la propaganda regia y los ámbitos religiosos y culturales, enfatizando la particular sonoridad del periodo medieval y renacentista y su intervención en la vida cotidiana de la sociedad. En opinión de Hablot y Vissière, el grito constituyó un elemento primordial en el paisaje sonoro, distinguiéndose distintos tipos: los gritos de la vida cotidiana, de las emociones y, el más emblemático de todos, el grito de guerra ${ }^{79}$.

Estas iniciativas francesas posibilitaron la apertura temática de los estudios medievales en otras latitudes. Un ejemplo a destacar ${ }^{80}$ es la producción del Grupo de Investigación y Estudios Medievales de la Universidad Nacional de Mar del Plata (Argentina) dedicada a la exploración y comprensión de la intervención de los sentidos - el auditivo en particular-en la configuración de la vida social a lo largo de la Edad Media: Paisajes sensoriales. Sonidos y silencios de la Edad Media ${ }^{81}$, Abordajes sensoriales del mundo medieval ${ }^{82}$ y Paisajes sonoros medievales $^{83}$ En ambas obras, investigadores latinoamericanos, estadounidenses, españoles y franceses exploraron diversos espacios europeos en pos de comprender cómo se conformaban los paisajes sonoros y cómo éstos mediaban en la aprehensión del mundo y en la construcción de los parámetros que regían la organización social y cultural.

77. L. Vissière, "Le paysage sonore parisien aux XIIIe et XIVe siècles ou la naissance”, Bulletin de la Société Nationale des Antiquaires de France, 2010, 136-158.

78. Vissière, "Le paysage sonore parisien...", op. cit., 139-143.

79. L. Hablot y L. Vissiere (dir.), Les paysages sonores du Moyen Âge à la Renaissance, Rennes, 2016, 12-15.

80. Un caso a destacar es la obra de Clara Bejarano Pellicer para el caso español en la Edad Moderna. En ella obtiene una representación sonora de la ciudad de Sevilla durante el Antiguo Régimen, analizando los sonidos del tiempo, de los ritos, de la recreación y las festividades sacras y profanas junto con aquellos sonidos identificadores de profesiones, como eran pregoneros y campaneros, desde una lectura musical de fuentes notariales y archivos civiles y eclesiásticos, C. Bejarano Pellicer, Los sonidos de la ciudad: el paisaje sonoro de Sevilla, siglos XVI al XVIII, Sevilla, 2015.

81. G. Rodríguez Gerardo y G. Coronado Schwindt (dirs.), Paisajes sensoriales. Sonidos y silencios de la Edad Media, Mar de Plata, 2016.

82. G. Rodríguez y G. Coronado Schwindt (dirs.), Abordajes sensoriales del mundo medieval, Mar del Plata, 2017.

83. G. Rodríguez Gerardo y G. Coronado Schwindt (dirs.), Paisajes sonoros medievales, Mar del Plata, 2019. 
Los paisajes sonoros medievales no sólo estuvieron integrados por sonidos que manifestaban el discurrir diario de los hombres y mujeres, sino también su desaparición. Murielle Gaude-Ferragu analizó un componente importante de la sonoridad en la Baja Edad Media, como fueron las expresiones de dolor en los rituales funerarios señaladas a través del grito; sones que fueron rechazados por la Iglesia al intentar imponer un comportamiento moderado con respecto al dolor. El autor se planteó determinar si los alaridos, en tanto claves espontáneas de emoción ante la muerte, eran una expresión ritual de sufrimiento en su estructura, intensidad y funcionalidad, mediante el análisis de distintas fuentes escritas (crónicas, relatos de funerales y los libros de contabilidad real). Además de los eventos de luto, estudió el papel de los pregoneros en las ceremonias funerarias reales. Contrariamente a los designios de la Iglesia, las manifestaciones de pena (lágrimas y gritos) eran frecuentes en los tiempos bajomedievales, puesto que fueron necesarias para exteriorizar el dolor popular que, a su vez, se traducía en cualidades humanas y políticas del señor. La profunda dolencia ritualizada y socializada, necesitó de los alaridos para legitimar a la persona fallecida, ostentando una función política en la sociedad ${ }^{84}$.

Todas estas iniciativas comprenden el imprescindible estado del arte de un campo de estudios que busca construir una Historia de los sentidos capaz de convertirse en una vía plausible de análisis de las sociedades actuales y pasadas. Esta posibilidad, sin embargo, no tiene por objetivo modificar las interpretaciones sobre lo acontecido. Por el contrario, procura texturizar, ahondar y complejizar cuestiones ya planteadas pero que, sin embargo, se podrían comprender e interrogar en un nivel más profundo, cumpliendo con ello uno de los principales propósitos de la investigación histórica: expandir el conocimiento de la experiencia humana.

\section{A modo de conclusión: ¿es posible un abordaje sensorial de la Edad Media?}

Mark Smith declaró en la revista Journal of Social History: «Es un buen momento para ser un historiador sensorial. La historia sensorial, también conocida como la historia de los sentidos (...), está en auge entre los historiadores ${ }^{85}$. Sin embargo, advirtió que se proyectaba en dos direcciones: en la historización de los sentidos y en la creación de un pasado sensorial ahistórico. Además de este último peligro, manifestó los problemas que se plantean en relación a la metodología y la presentación de estos enfoques, debido, en parte, a la misma velocidad con la que la Historia de los sentidos había ganado terreno ${ }^{86}$. Por ello, una aproximación a la dimensión sensorial de las sociedades premodernas conlleva la difi-

84. M. Gaude-Ferragu, "Le cri dans le paysage sonore de la mort à la fin du Moyen Âge", en D. Lett y N. Offenstadt (dirs.), Haro! Noël! Oyé!: pratiques du cri au Moyen Âge, Paris, 2003, 93-102.

85. «It is a good moment to be a sensory historian. Sensory history-also referred to as the history of the senses (...) —is booming among historians», M. Smith, "Producing sense, consuming sense, marking sense: perils y prospects for Sensory History”, Journal of Social History, 40, 2007, 841-858. Traducción propia.

86. M. Smith, “Producing sense, consuming...”, op. cit., 841. 
cultad de examinar registros que no han sido creados con la intención de transmitir marcas sensoriales ${ }^{87}$. Es aquí donde radica la habilidad del investigador para leer entre líneas, buscando las significaciones sensoriales de las prácticas y los espacios por medio del armado de un marco teórico-metodológico interdisciplinar, que por norma no se encuentran de forma innata en una sola disciplina social ${ }^{88}$.

A lo largo de estas páginas analizamos los estudios que, en nuestra opinión, son los más relevantes en los estudios históricos sobre los sentidos y, en particular, los interesados en las percepciones sensoriales de los tiempos medievales. En este estudio destacamos no solo su temática específica, sino también los postulados teóricos y metodológicos generales que sostienen sus investigaciones y las dificultades que tuvieron que sortear. Los obstáculos más evidentes son la brevedad de las pruebas presente en los registros documentales y el peligro del uso de la metáfora en las fuentes narrativas. En todos estos análisis, el hilo conductor que los posiciona en el campo de los estudios sensoriales es reconocer la naturaleza histórica y cultural de los sentidos, destacando su participación en la construcción de los parámetros políticos, sociales y culturales de la sociedad medieval.

La pesquisa historiográfica que realizamos nos permitió exponer los primeros intentos de captar la materia sensible de las sociedades del pasado, reconociendo en las figuras de Huizinga y Febvre sus principales precursores. La década de los ochenta representó el comienzo de la exploración de los sentidos en materia histórica y antropológica, enfatizando desde el principio, la necesidad de entablar una verdadera interdisciplinariedad. En nuestra opinión, Alain Corbin, Constance Classen y David Howes, son los principales constructores de este campo. En particular, la Edad Media ha comenzado a ser revisitada en función de este nuevo enfoque. Conocer a los hombres y mujeres medievales a través de lo que vieron, escucharon, olieron, degustaron y tocaron, y cómo esa información codificó su mundo, se volvió una vía plausible de análisis. En este marco, destacamos tres perspectivas generales desde las que se abordaron los sentidos. En una primera instancia, fue el arte el vehículo de observación, destacándose los estudios sobre la representación pictórica de los sentidos, ya sea por medio del simbolismo animal o de operaciones metafóricas que representaban una percepción. En una segunda instancia, la liturgia, los manuales de confesión, los penitenciales medievales y la literatura en general, y el interés por la actuación de la sensorialidad en la cultura cristiana medieval, fueron el punto central del abordaje sensorial. Y por último, el análisis de los sentidos se ha centrado en el marco de la vida cotidiana, los objetos y los espacios habitados, in-

87. Con la noción "marcas sensoriales" (visuales, auditivas, olfativas, gustativas y táctiles) los autores identifican a las percepciones que guardan una especial significación para la trama de una cultura. Este concepto hace referencia a las soundmarks formuladas por Raymond Murray Schafer, con las cuales reconoce a todos aquellos sonidos que revisten importancia para una sociedad, de acuerdo con el valor simbólico y afectivo que poseen, G. Rodríguez y G. Coronado Schwindt, "La intersensorialidad en el Waltharius", Cuadernos Medievales, 23, 2017, 31-48.

88. C. Astarita, "Historia y ciencias sociales. Préstamos y reconstrucción de categoría analíticas", Sociohistórica, 8, 2000, 28. 
vestigaciones que han destacado la importancia de la experiencia sensorial en el devenir diario desde una doble perspectiva: en la aprehensión y decodificación de su realidad y, simultáneamente, en la construcción de sentidos de esa realidad. De forma particular, la Edad Media ha sido revisitada por medio de la noción de «paisaje sonoro», logrando con ello identificar y comprender los sonidos y silencios que conformaron y dieron forma a la sociedad medieval.

En definitiva, el futuro de los estudios sensoriales centrados en la Edad Media es prometedor y día a día se consolida entre los historiadores. El paso determinante en los años venideros será el de transformar este «campo» en un «hábito». Por «campo» se concibe al ámbito académico delimitado de la investigación, con conciencia de su propia existencia y sus propios imperativos. En cambio, por «hábito» se entiende a la forma de examinar el pasado, desde postulados metodológicos y epistemológicos, que permitan identificar e interpretar las pistas sensoriales presentes en las fuentes ${ }^{89}$. Alcanzar este estadio de comprensión de la sensorialidad en la investigación histórica es una de las metas que se han trazado los investigadores en la actualidad, en consonancia con el deseo de conocer y aprehender a los sujetos históricos en sus prácticas intrínsecas de la percepción de su mundo.

89. M. Smith, "Futures of hearing pasts", en D. Morat (ed.), Sounds of Modern History: Auditory Cultures in 19th and 20th Century Europe, New York-Oxford, 2014, 13-14. 